\title{
Prediction and Exploitation of Transformation Texture
}

\author{
BHADESHIA Harshad K. D. H. \\ University of Cambridge, Materials Science and Metallurgy, U. K.
}

hkdb@cam.ac.uk

Keywords: Crystallography, transformation texture, martensite, bainite, allotriomorphic ferrite, steel

\begin{abstract}
There is now a great deal known about the atomic mechanisms of solid-state phase transformations, and this knowledge can be exploited to determine the distribution of crystal orientations. It is possible to estimate accurately, the crystallographic texture, transformation strains and details of the microstructure, particularly in the context of steels. The concepts have now been applied to design metallic alloys which compensate automatically for the residual stresses which develop in engineering components when they are cooled heterogeneously from elevated temperatures. Such materials are now in commercial use and represent an innovation resulting directly from phase transformation theory.
\end{abstract}

\section{Introduction}

A polycrystalline material is said to be crystallographically textured when the distribution of crystal orientations relative to an external frame of reference is non-random. Texture can arise for many reasons, for example, plastic and elastic deformation, magnetic fields, recrystallisation, etc. but the focus of the present paper is on phase transformations.

When a crystal forms in the solid-state, constrained by the surrounding parent phase, it tends to do so in a way which minimises the excess free energy. The process may involve the development of an orientation relationship which minimises the total interfacial and elastic strain energies. Or alternatively, there may exist constraints on atomic mobility which control the crystallography of transformation and place restrictions on the nature of interfaces that can or cannot exist. To consider this problem in detail it is necessary to define the mechanisms of transformation on the basis of evidence, summarised in Fig. 1. A detailed explanation can be found elsewhere [1] but it is important for the present purpose to recognise that there are transformations which produce displacements consistent with the crystallographic theory of martensite $[2,3,4]$. These are the displacive transformations which result in a shape deformation that is an invariant-plane strain with a large shear component and a dilatation normal to the habit plane of the plate-shaped product that grows. The reconstructive transformations on the other hand can have arbitrary morphology and their growth involves diffusion fluxes which do not result in a shape deformation other than the minor component that is the change in density on transformation.

One consequence is that the displacive product is confined to the austenite grain in which it nucleates, because the synchronised movement of atoms during growth cannot be sustained across a grain boundary. In contrast, a reconstructive product is built up by a random motion of atoms by diffusion and hence is not limited by the grain boundaries of the parent phase, Fig. 2.

We now proceed to explain how the texture might be calculated for both kinds of transformation mechanisms. 


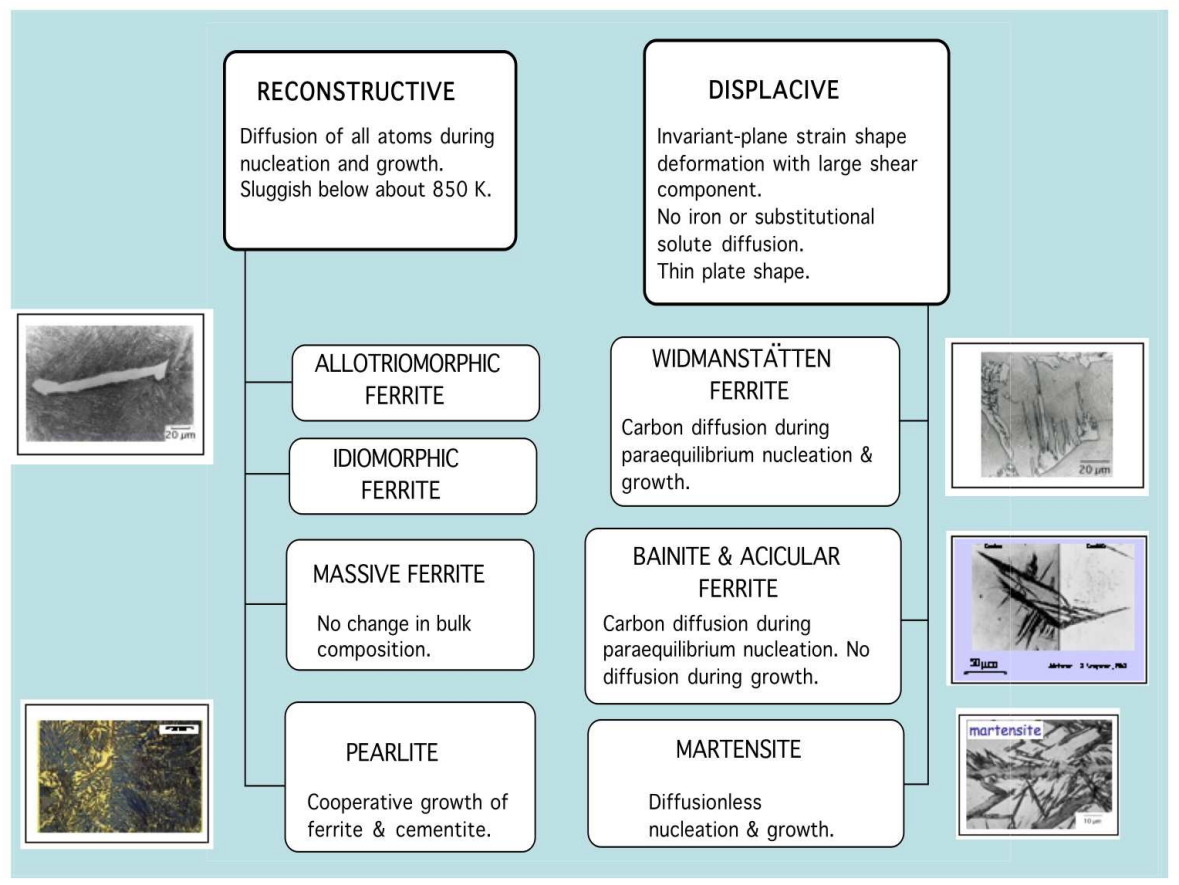

Fig. 1: Summary of the transformation mechanisms for some of the key transformation products of austenite. The scheme is based on a consistent interpretation of a large amount of experimental and theoretical evidence as described in $[1,5]$.

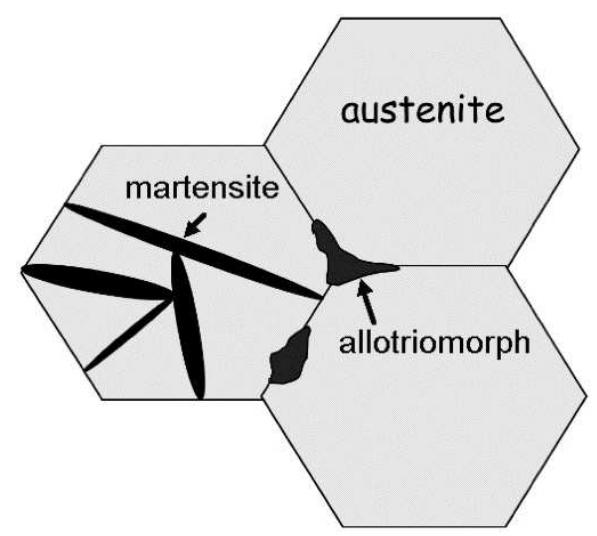

Fig. 2: An illustration of the fact that martensite, bainite and Widmanstätten ferrite, all grow in the form of plates which do not cross austenite $(\gamma)$ grain boundaries because it is not possible to sustain a disciplined motion of atoms through a change in $\gamma$-grain orientation. In contrast, the growth of allotriomorphic ferrite or pearlite occurs by a reconstructive mechanism involving long-range diffusion so that the growing particle is not confined to the grain in which it nucleates.

\section{Displacive Transformations}

The need to minimise strain energy requires in this case that the product is in the form of a thin plate [6]. This in turn leads to three unique characteristics associated with each crystal which forms in a given austenite grain:

- the habit plane on which each plate grows, i.e., the major interface between the parent and product lattices - in general has irrational indices;

- there exists a strictly respected orientation relationship, which in general is irrational;

- there is a deformation caused by transformation, described as an invariant-plane strain shape with a large shear component and a dilatation normal to the habit plane.

It is important to realise that these three features are mathematically connected and cannot be varied independently. This is in contrast to the general description of a bicrystal, defined by five degrees of freedom, two for the interfacial plane and three for the relative orientation, as 
illustrated in Fig. 3. In that illustration, the interface plane can be altered without changing the orientation relationship, and vice-versa. The reason for the connections implicit in displacive transformation products are described in the next section.

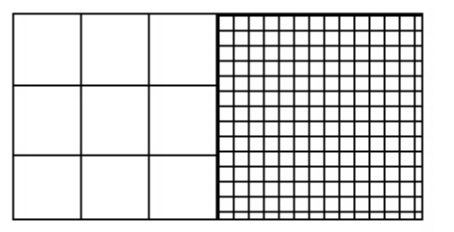

\section{Reference bicrystal}

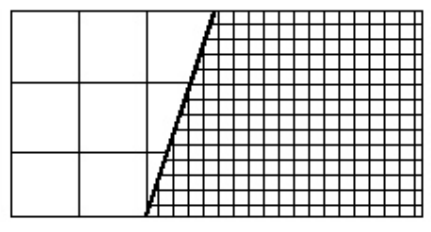

Orientations fixed, interface plane altered

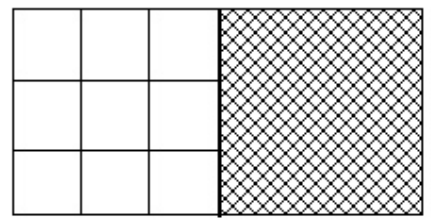

Orientation altered, interface plane fixed
Fig. 3: An illustration of the fact that the orientation relationship between crystals can be independently varied without altering the plane of the interface and vice-versa. If the two crystals belong to same crystal class (of which there are seven) then the orientation relation can be described by an axis and an angle of rotation about that axis. The interface plane is specified by an axis normal to the plane. Since each axis can be written as a unit vector we need specify only two of its components. Thus, the total degrees of freedom required to specify the bicrystal is five.

\section{Crystallographic Theory}

When austenite ( $\gamma$, cubic-close packed crystal structure) transforms into martensite ( $\alpha^{\prime}$, bodycentred cubic or body-centred tetragonal), bainite or Widmanstätten ferrite, the change in crystal structure is achieved by a homogeneous deformation known as the Bain strain $\mathbf{B}$. The Bain correspondence is illustrated in Fig. 4 but the orientation illustrated is not observed experimentally. The deformation also does not satisfy the minimum requirement of displacive transformation, that at least one line must be left invariant to ensure sufficient coherency in the $\gamma / \alpha^{\prime}$ interface so that it can move without a need for diffusion. This can be seen in Figs. 5a, b; the austenite is represented as a sphere which, as a result of the Bain strain $\mathbf{B}$, is deformed into an ellipsoid of revolution which represents the martensite. There are no lines which are left undistorted or unrotated by $\mathbf{B}$. There are no lines in the $\left(\begin{array}{lll}0 & 0 & 1\end{array}\right)_{\gamma}$ plane which are undistorted. The lines $a b$ and $c d$ are undistorted but are rotated to the new positions $a^{\prime} b^{\prime}$ and $c^{\prime} d^{\prime}$. Such rotated lines are not invariant. However, the combined effect of the Bain strain $\mathbf{B}$ and the rigid body rotation $\mathbf{R}$ is indeed an invariant-line strain (ILS) because it brings $c d$ and $c^{\prime} d^{\prime}$ into coincidence (Fig. 5c). This is why the observed irrational orientation relationship differs from that implied by the Bain strain. Indeed, the rotation required to convert $\mathbf{B}$ into an invariant line strain precisely corrects the Bain orientation into that which is observed experimentally.

As can be seen from Fig. 5, there is no rotation which can make $\mathbf{B}$ into an invariant-plane strain since this would require two non-parallel invariant-lines. It follows that austenite cannot be transformed into martensite by an invariant-plane strain. And yet, the observed shape deformation leaves the habit plane undistorted and unrotated, i.e., it is an invariant-plane strain.

The phenomenological theory of martensite crystallography solves this remaining problem (Fig. 6). The Bain strain converts the structure of the parent phase into that of the product 


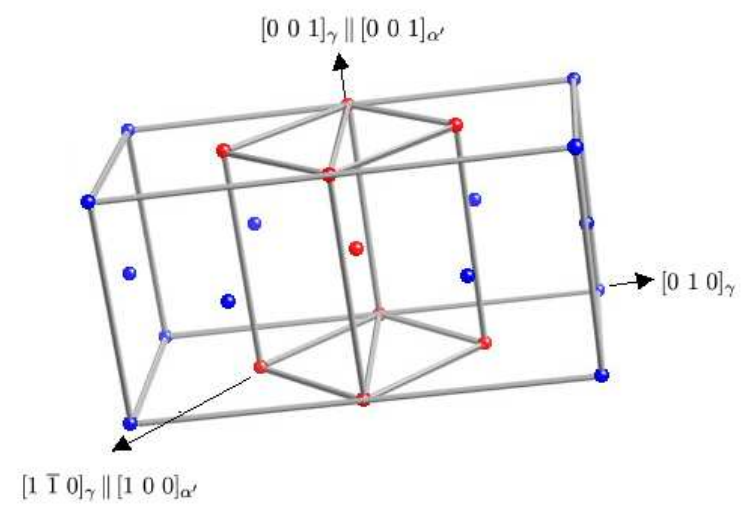

Fig. 4: Two face-centred cubic unit cells of austenite, together with a body-centred tetragonal cell of austenite. The Bain strain (not illustrated here) involves a compression of the body-centred tetragonal cell of austenite along $[001]_{\gamma}$ and a uniform expansion on the $(001)_{\gamma}$ plane.

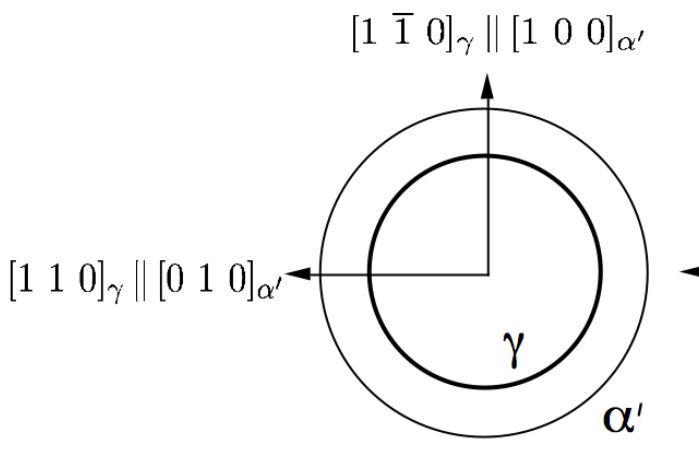

(a)

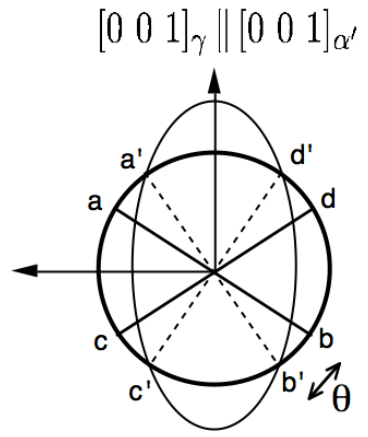

(b)

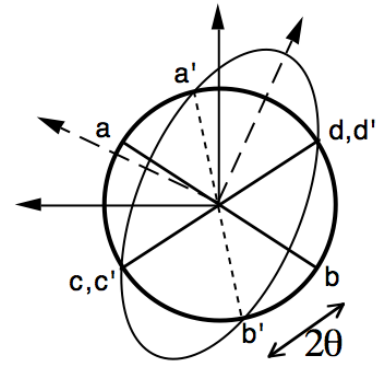

(c)

Fig. 5: (a) and (b) show the effect of the Bain strain on austenite, which when undeformed is represented as a sphere of diameter $a b=c d$ in three-dimensions. The strain transforms it into an ellipsoid of revolution. (c) shows the ILS obtained by combining the Bain strain with a rigid body rotation through an angle $\theta$.

phase. When combined with an appropriate rigid body rotation, the net homogeneous lattice deformation RB is an invariant-line strain (step $a$ to $c$ in Fig. 6). However, the observed shape deformation is an invariant-plane strain $\mathbf{P}_{\mathbf{1}}$ (step $a$ to $b$ ), but this gives the wrong crystal structure. If a second homogeneous shear $\mathbf{P}_{\mathbf{2}}$ is combined with $\mathbf{P}_{\mathbf{1}}$ (step $b$ to $c$ ), then the correct structure is obtained but the wrong shape since

$$
\mathrm{P}_{1} \mathrm{P}_{2}=\mathrm{RB}
$$

These discrepancies are all resolved if the shape changing effect of $\mathbf{P}_{\mathbf{2}}$ is cancelled macroscopically by an inhomogeneous lattice-invariant deformation, which may be slip or twinning.

The theory explains all the observed features of the martensite crystallography. The orientation relationship is predicted by deducing the rotation needed to change the Bain strain into an invariant-line strain. The habit plane does not have rational indices because the amount of lattice-invariant deformation needed to recover the correct the macroscopic shape is not usually rational. The theory predicts a substructure in plates of martensite (either twins or slip steps) as is observed experimentally. The transformation goes to all the trouble of ensuring that the shape deformation is macroscopically an invariant-plane strain because this reduces the strain 
energy when compared with the case where the shape deformation might be an invariant-line strain.

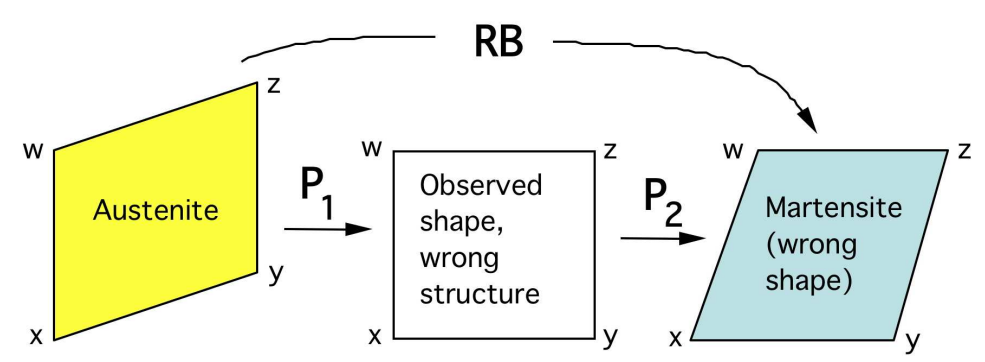

(a)

(b)

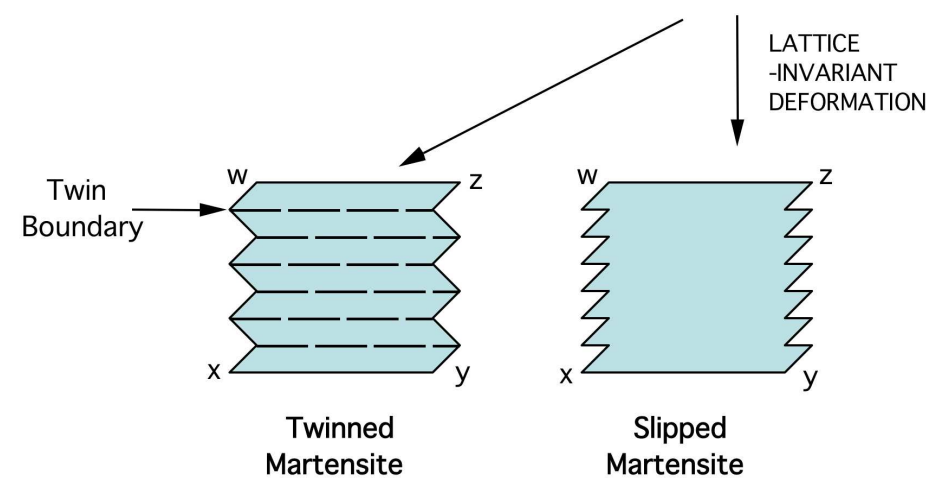

Fig. 6: The crystallographic theory of martensitic transformation.

Correct macroscopic shape, correct structure

Given the tenets of the crystallographic theory via equation 1, the information required to describe a single plate of martensite, bainite or Widmanstätten ferrite is similar to the data listed in Table 1.

Table 1: Typical crystallographic set for displacive transformation for $a_{\gamma} / a_{\alpha^{\prime}}=1.2557$ [7].

$$
\begin{aligned}
& \text { Habit plane } \mathbf{p}_{\gamma} \\
& \text { Shape change }(\gamma \mathrm{P} \gamma) \\
& \left(\begin{array}{l}
-0.168640 \\
-0.760394 \\
-0.627185
\end{array}\right) \\
& \left(\begin{array}{ccc}
0.992654 & -0.033124 & -0.027321 \\
0.026378 & 1.118936 & 0.098100 \\
-0.027321 & -0.123190 & 0.898391
\end{array}\right) \\
& \left(\begin{array}{ccc}
0.575191 & 0.542067 & 0.097283 \\
-0.550660 & 0.568276 & 0.089338 \\
-0.008610 & -0.131800 & 0.785302
\end{array}\right) \quad\left[\begin{array}{lll}
1 & 0 & 1
\end{array}\right]_{\gamma} \|[-0.920611-1.0626371 .084959]_{\alpha^{\prime}}
\end{aligned}
$$

\section{Using the Crystallographic Set}

Displacive transformations cause deformation so those crystallographic variants which best comply with external stress will be favoured. The deformation occurs on the habit plane (unit 
normal $\mathbf{p}$ ) in a displacement direction (unit vector $\mathbf{d}$ ). The dominant strain is the shear $(s)$ parallel to the habit plane which in steels is about 0.26 with the dilatational strain $(\delta)$ being of the order of 0.03. The total deformation is expressed as a $3 \times 3$ matrix $\mathbf{P}$ :

$$
(\gamma \mathrm{P} \gamma)=\mathrm{I}+m[\gamma ; \mathbf{d}]\left(\mathbf{p} ; \gamma^{*}\right)
$$

where $m$ is the magnitude of the shape deformation and $\gamma$ and $\gamma^{*}$ represent the real and reciprocal bases of the austenite. The matrix $\mathbf{P}$ thus completely defines the deformation system. Note that there will always be 24 different variants since the orientation relationship is irrational. The mechanical free energy due to the interaction of the stress with the shape deformation is given by [8]:

$$
U=\sigma_{N} \zeta+\tau s
$$

rigourous where $\sigma_{N}$ is the stress component normal to the habit plane, $\tau$ is the shear stress resolved on the habit plane in the direction of shear and $\zeta$ and $s$ are the respective normal and shear strains associated with transformation. The energy $U$ can be used as a rigorous variant selection criterion when the stresses applied are less than those required to cause plasticity in the austenite prior to its transformation [9, 10, 11, 12, 13].

The conclusions that can be reached from the discussion in this section are:

- In calculating transformation texture is is necessary to use a a self-consistent crystallographic set, rather than make independent assumptions about the orientation relationship and shape deformation as is sometimes done. The set must be such that the lattice deformation BR is an invariant-line strain; the analysis in [14] does not satisfy this criterion.

- The deformation due to martensitic transformation is an invariant-plane strain $\mathbf{P}$. It is this which should be used to calculate the interaction energy (variant selection) rather than, for example, the Bain strain [15].

\section{Transformation Plasticity}

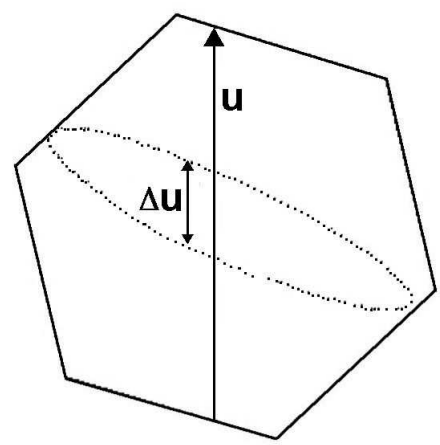

(a)

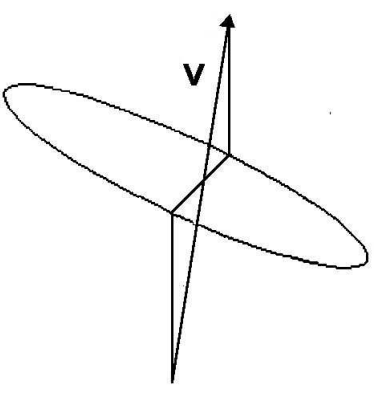

(b)

Fig. 7: The deformation of an initial vector $\mathbf{u}$ by the formation of bainite. (a) An austenite grain prior to transformation, with the ultimate location of a plate of bainite marked. (b) Following displacive transformation. [11]

Consider an arbitrary vector $\mathbf{u}$ traversing a grain of austenite prior to transformation, as illustrated in Fig. 7a. This vector makes an intercept $\Delta \mathbf{u}$ with a domain of austenite that 
eventually ends up as a plate of bainite. As a consequence of the transformation, the vector $\mathbf{u}$ becomes a new vector $\mathbf{v}$ given by [11]:

$$
\mathbf{v}=\mathbf{P} \Delta \mathbf{u}+(\mathbf{u}-\Delta \mathbf{u})
$$

When considering the formation of large number of bainite plates in many austenite grains, $\mathbf{u}$ traverses a polycrystalline sample of austenite so this equation must be generalised as follows [11]:

$$
\mathbf{v}=\sum_{k=1}^{n} \sum_{j=1}^{24} \mathbf{P}_{j}^{k} \Delta \mathbf{u}_{j}^{k}+\left(\mathbf{u}-\sum_{k=1}^{n} \sum_{j=1}^{24} \Delta \mathbf{u}_{j}^{k}\right)
$$

where $j=1 \ldots 24$ represents the 24 crystallographic variants possible in each austenite grain, and $k=1 \ldots n$ represents the $n$ austenite grains traversed by the vector $\mathbf{u}$. In this scenario of a large number of bainite plates, the intercepts $\Delta \mathbf{u}_{j}^{k}$ can be approximated by $f_{j}^{k} \mathbf{u}$ where $f_{j}^{k}$ is the fraction of sample transformed by variant $j$ in austenite grain $k$.

The deformation caused by a particular plate $j$ in austenite grain $k$, i.e., $\left(\gamma_{k} \mathrm{P}_{\mathrm{j}} \gamma_{k}\right) \equiv$ $\mathbf{P}_{j}^{k}$. The remaining 23 such matrices for grain 1 of austenite can be deduced from this using symmetry operations. They can then be expressed in the reference frame of the sample using a similarity transformation as follows:

$$
\left(\mathrm{S} \mathrm{P}_{j}^{k} \mathrm{~S}\right)=\left(\mathrm{S} \mathrm{R} \gamma_{k}\right)\left(\gamma_{k} \mathrm{P}_{j} \gamma_{k}\right)\left(\gamma_{k} \mathrm{R} \mathrm{S}\right)
$$

where $\left(\mathrm{SR} \gamma_{k}\right)$ is the rotation matrix relating the basis vectors of the $k$ th austenite grain to the sample axes, and $\left(\gamma_{k} \mathrm{R} S\right)$ is the inverse of that rotation matrix. In this way, the calculation described in equation 4 can be conducted in the sample frame of reference.

Some calculations illustrating the anisotropy of strains as a function of the number of crystallographic variants of martensite allowed are presented in Fig. 8 for pure shear and compression. That displacive transformations produce highly anisotropic strains when variant selection is significant has been demonstrated experimentally $[16,17,18]$. The mechanism by which a polycrystal of austenite behaves in this manner is illustrated in Fig. 9.

(a)

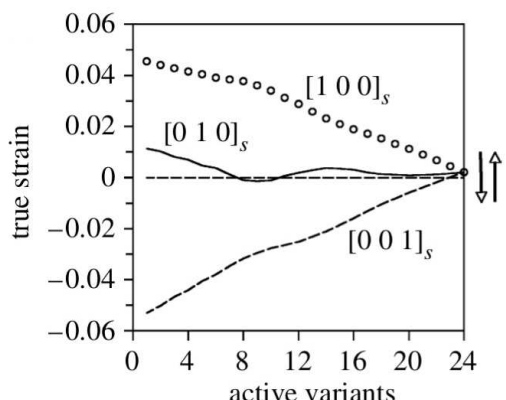

(b)

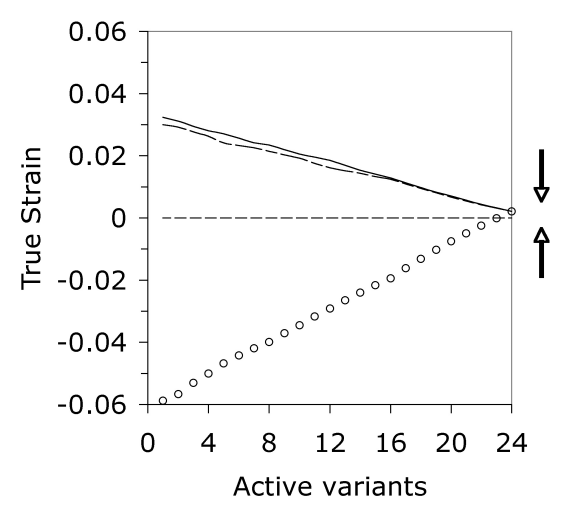

Fig. 8: to transformation along the $\left[\begin{array}{lll}1 & 0 & 0\end{array}\right]_{S}$ direction (labelled longitudinal, along the stress axis), and the transverse directions $\left[\begin{array}{lll}0 & 1 & 0\end{array}\right]_{S}$ and $\left[\begin{array}{lll}0 & 0 & 1\end{array}\right]_{S}$. (a) Pure shear. (b) Compressive stress [11]d.

An important outcome of the analysis is that the method not only predicts the crystallographic orientations to be expected $[7,19,11,20]$ but also the transformation strains and the specific orientations of the habit planes of martensite. There is often a distinction made in the literature between stress-induced and strain-induced transformation, where the former 

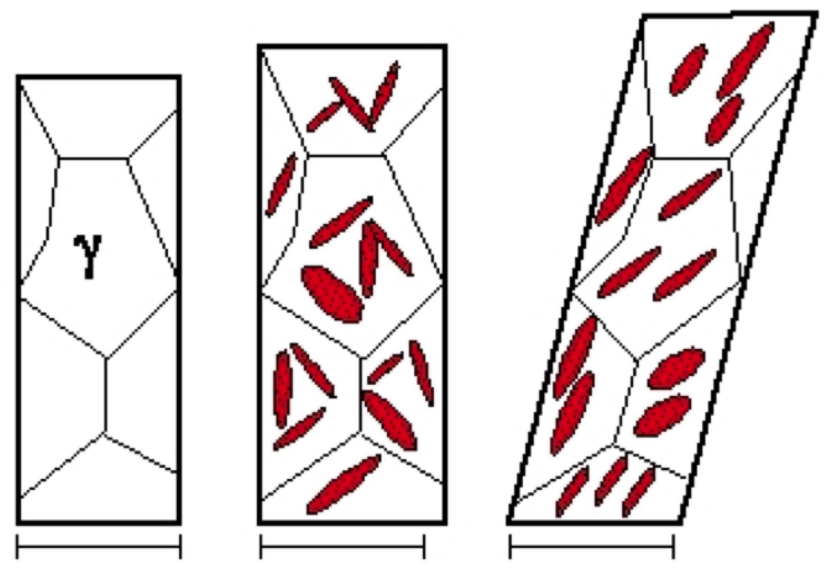

Fig. 9: (a) Polycrystaline austenite. (b) Polycrystalline austenite partially transformed into plates of martensite which on a macroscopic scale are randomly dispersed so that an isotropic volume change is observed. (c) Polycrystalline austenite in which variants which comply with an external stress are favoured. The transformation strains now become anisotropic.

is driving by the mechanical free energy embodied in equation 3, and the latter by the introduction of nucleating defects within the austenite. However, the vast majority of experiments designed to study strain-induced transformation are conducted where the stress and strain are simultaneously applied; such experiments can for reasonably large strains, be explained purely on the basis of the mechanical driving force associated with stress-affected transformation $[9,7,10,12,13,21]$. This makes it easier to calculate the orientations of the transformation products because the theory for strain-induced transformation is not as clear because it requires a relationship to be established between the number density and potency of additional nucleation sites introduced by deformation.

One example of the comparison between calculated and measured transformation textures is illustrated in Fig. 10, with the calculations being conducted both with and without variant selection. The former is clearly more representative of the measured texture, although it should be borne in mind that the comparison with experimental data is solely in the prediction of orientation rather than intensity of orientation.

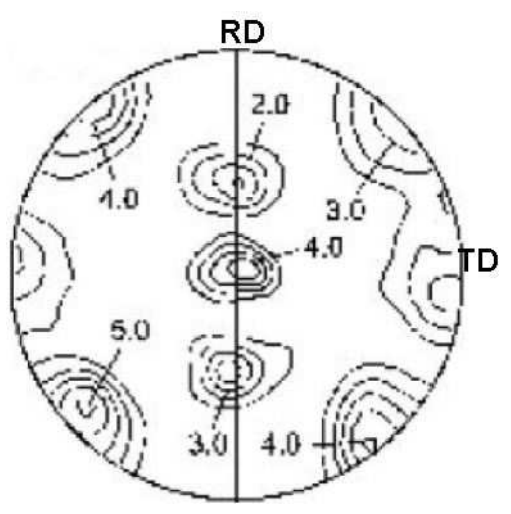

(a)

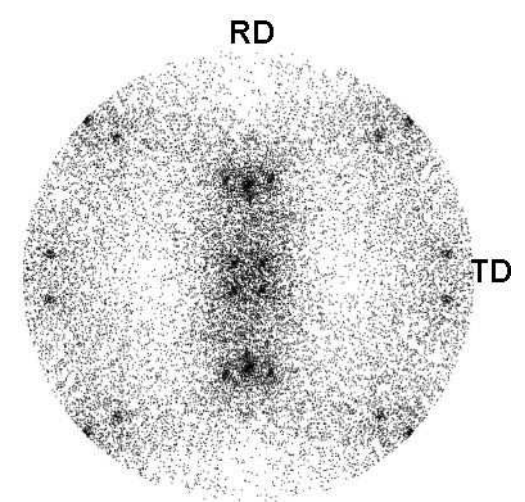

(b)

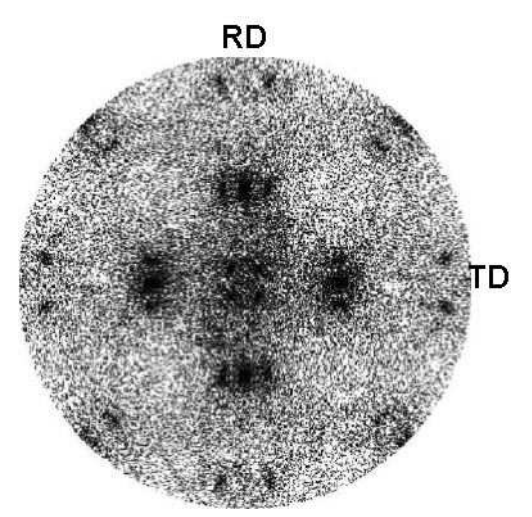

(c)

Fig. 10: $100_{\alpha^{\prime}}$ pole figures for transformation of Cube oriented austenite grains. (a) Experimental data [22]. (b) Calculations allowing only favoured variants of martensite to form in each austenite grain. (c) Calculations allowing all possible variants of martensite to form in each austenite grain. 


\section{Application}

Residual stresses due to transformations are often introduced unintentionally during fabrication [23, 23]. Jones and Alberry [24] conducted experiments to illustrate the role of transformations on the development of residual stress in steels. Using bainitic, martensitic and stable austenitic steels, they demonstrated that transformation plasticity during the cooling of a uniaxially constrained sample from the austenite phase field, acts to relieve the build up of thermal stress as the sample cools. In contrast, the non-transforming austenitic steel exhibited a monotonic increase in residual stress with decreasing temperature, as might be expected from the thermal contraction of a constrained sample. When the steels transformed to bainite or martensite, the transformation strain compensated for any thermal contraction strains that arose during cooling. Significant residual stresses were therefore found to build up only after transformation was completed, and the specimens approached ambient temperature. Fig. 11 illustrates the sequence of events when a specimen constrained when it is fully austenitic, is cooled continuously. It is evident that is evident that the transformation plasticity due to variant selection plays a key role in compensating for the development of stress, but the stress-state at ambient temperature is non-zero if the transformation is exhausted before it has cooled completely.

The key therefore is to design welding alloys in which the transformation becomes exhausted only when ambient temperature is reached. This is a large and exciting subject and the reader is referred to an extensive literature which includes major reviews [24, 25, 26, 27, 23, 28, 29, $30,31,32,33,34,35,36,37,38]$.

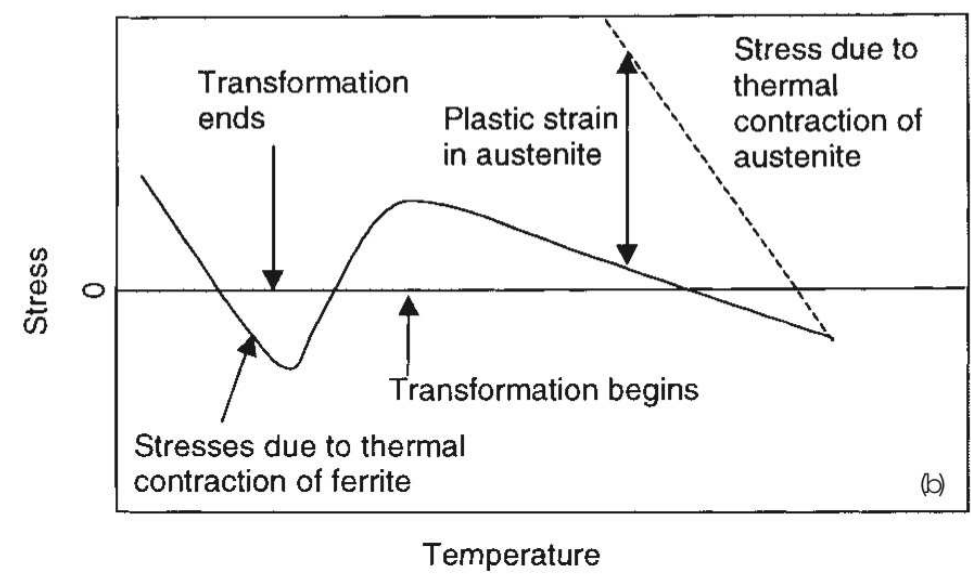

Fig. 11: Variation in stress when a sample of austenite is allowed to cool whilst being constrained. The initial rise is stress is controlled by thermal contraction, followed by compensation due to transformation plasticity.

\section{Reconstructive Transformations}

Reconstructive transformations are not confined to the grains in which they nucleate, they may form in contact with 1-4 austenite grains depending on the exact location of the nucleus, and the interfaces can be varied independently of the orientation relationship.

The following excerpt is based on a review [39], and highlights the fact that the number of degrees of freedom available to form crystal orientation relationships becomes unmanageable when dealing with reconstructive transformations in polycrystalline austenite:

"The number of parameters required to describe the locations of $N$ crystals in an aggregate with respect to a frame of reference is $3 N$. An equiaxed grain in the form of a Kelvin tetrakaidecahedron will have fourteen faces, so that the number of bicrystal orientations that must be described per grain becomes $\frac{1}{2} \times 14 \times 3 N=21 N$. Each of these interfaces will have two degrees of freedom so the parameters become $14 \times 2 \times 21 N=588 N$. A typical grain size is about $10 \mu \mathrm{m}$ so a cubic centimetre of material will contain $N=10^{12}$ grains so that its full description 
requires about $10^{15}$ parameters! If the volume of each grain also needs to be specified than the problem become intractable."

\section{Conclusions}

There is limited progress in the calculation of transformation textures. In the case of displacive reactions, it is possible to apply the crystallographic theory of martensite combined with the concept of mechanical driving force to calculate the product orientations that develop from a known set of parent orientations. It is not, however, possible to predict the expected intensities in pole figures or orientation distribution functions. This requires a coupling of the crystallographic theory with kinetic models. This has yet to be done.

The results of such analyses, given that they include both habit plane and transformation strain data, can and are being exploited in the design of commercial welding alloys.

Reconstructive transformations have so much more freedom to develop that the number of parameters that must be included in any theory is daunting. For this reason, it is likely that the gap between experiments and theory will remain large for the foreseeable future.

\section{References}

[1] H. K. D. H. Bhadeshia: Bainite in Steels, 2nd edition: Institute of Materials, London, 2001.

[2] J. S. Bowles, J. K. Mackenzie: Acta Metallurgica Vol. 2 (1954) p. 129.

[3] J. K. Mackenzie, J. S. Bowles: Acta Metallurgica Vol. 2 (1954) p. 138.

[4] M. S. Wechsler, D. S. Lieberman, T. A. Read: Trans. AIME Journal of Metals Vol. 197 (1953) p. 1503.

[5] H. K. D. H. Bhadeshia, J. W. Christian: Metallurgical \& Materials Transactions A Vol. 21A (1990) p. 767.

[6] J. W. Christian: Acta Metallurgica Vol. 6 (1958) p. 377.

[7] S. Kundu, H. K. D. H. Bhadeshia: Scripta Materialia Vol. 55 (2006) p. 779.

[8] J. R. Patel, M. Cohen: Acta Metallurgica Vol. 1 (1953) p. 531.

[9] I. Tamura: Metal Science Vol. 16 (1982) p. 245.

[10] S. Chatterjee, H. K. D. H. Bhadeshia: Materials Science and Technology Vol. 23 (2007) p. 1101.

[11] S. Kundu, K. Hase, H. K. D. H. Bhadeshia: Proceedings of the Royal Society A Vol. 463 (2007) p. 2309.

[12] E. S. Perdahcioüglu, H. J. M. Geijselaers, M. Groen: Scripta Materialia Vol. 58 (2008) p. 947.

[13] H. J. M. Geijselaers, E. S. Perdahciouglu: Scripta Materialia Vol. 60 (2009) 29.

[14] D. W. Suh, H. N. Han, S. J. Kim: ISIJ International Vol. 46 (2006) p. 341.

[15] H. N. Han, D. W. Suh: Acta Materialia Vol. 51 (2003) p. 4907.

[16] H. K. D. H. Bhadeshia, S. A. David, J. M. Vitek, R. W. Reed: Materials Science and Technology Vol. 7 (1991) p. 686.

[17] A. Matsuzaki, H. K. D. H. Bhadeshia, H. Harada: Acta Metallurgica and Materialia Vol. 42 (1994) p. 1081. 
[18] H. K. D. H. Bhadeshia: Possible effects of stress on steel weld microstructures: in: H. Cerjak, H. K. D. H. Bhadeshia (Eds.), Mathematical Modelling of Weld Phenomena - II: Institute of Materials, London, U.K., 1995: pp. 71.

[19] S. Kundu: Transformation strain and crystallographic texture in steels: Ph.D. thesis: http://www.msm.cam.ac.uk/phase-trans/2000/phd.html\#kundu, University of Cambridge: Cambridge, U. K. (2007).

[20] M. Kundu, S. Ganguly, S. Datta, P. P. Chattopadhyay: Materials and Manufacturing Processes Vol. 24 (2009) p. 169.

[21] A. A. Shirzadi, H. Abreu, L. Pocock, D. Klobcar, p. J. Withers, H. K. D. H. Bhadeshia: International Journal of Materials Research Vol. 100 (2009) 40.

[22] N. Gey, B. Petit, M. Humbert: Metallurgical \& Materials Transactions Vol. 36 (2005) p. 3291.

[23] P. J. Withers, H. K. D. H. Bhadeshia: Materials Science and Technology Vol. 17 (2001) p. 355.

[24] W. K. C. Jones, P. J. Alberry: Metals Technology Vol. 11 (1977) p. 557.

[25] A. Ohta, N. Suzuki, Y. Maeda, K. Hiraoka, T. Nakamura: International Journal of Fatigue Vol. 21 (1999) p. S113.

[26] A. Ohta, O. Watanabe, K. Matsuoka, C. Shiga, S. Nishijima, Y. Maeda, N. Suzuki, T. Kubo: Welding in the World Vol. 43 (1999) p. 38.

[27] A. Ohta, N. Suzuki, Y. Maeda: in: A. Meike (Ed.), Properties of Complex Inorganic Solids 2: Kluwer Academic/Plenum Publishers, 2000: p. 401.

[28] p. J. Withers, H. K. D. H. Bhadeshia: Materials Science and Technology Vol. 17 (2001) p. 366.

[29] A. Ohta, K. Matsuoka, N. T. Nguyen, Y. Maeda, N. Suzuki: Welding Journal, Research Supplement Vol. 82 (2003) p. 77s.

[30] J. Eckerlid, T. Nilsson, L. Karlsson: Science and Technology of Welding and Joining Vol. 8 (2003) p. 353.

[31] H. Lixing, W. Dongpo, W. Wenxian, Y. Tainjin: Welding in the World Vol. 48 (2004) p. 34.

[32] S. Zenitani, N. Hayakawa, J. Yamamoto, K. Hiraoka, Y. Morikage, T. Yauda, K. Amano: Science and Technology of Welding and Joining Vol. 12 (2007) 516.

[33] J. A. Francis, H. J. Stone, S. Kundu, R. B. Rogge, H. K. D. H. Bhadeshia, P. J. Withers, L. Karlsson: Transformation temperatures and welding residual stresses in ferritic steels: in: Proceedings of PVP2007, ASME Pressure Vessels and Piping Division Conference: American Society of Mechanical Engineers: ASME, San Antonio, Texas, 2007: p. 1.

[34] P. P. Darcis, H. Katsumoto, M. C. Payares-Asprino, S. Liu, T. A. Siewert: Fatigue and Fracture of Engineering Materials and Structures Vol. 31 (2008) 125.

[35] M. C. Payares-Asprino, H. Katsumoto, S. Liu: Welding Journal, Research Supplement Vol. 87 (2008) p. 279s.

[36] H. Dai, J. A. Francis, H. J. Stone, H. K. D. H. Bhadeshia, P. J. Withers: Metallurgical \& Materials Transactions A Vol. 39 (2008) p. 3070.

[37] Y. Mikami, Y. Morikage, M. Mochizuki, M. Toyoda: Science and Technology of Welding and Joining Vol. 14 (2009) p. 97. 
[38] A. A. Shirzadi, H. K. D. H. Bhadeshia, L. Karlsson, P. J. Withers: Science and Technology of Welding and Joining Vol. 14 (2009) p. 559.

[39] H. K. D. H. Bhadeshia: ISIJ International Vol. 50 (2010) submitted. 